\title{
La contribución de la Unión Europea al desarrollo del régimen internacional en materia de cambio climático: el paquete europeo sobre clima y energía en el contexto de la acción internacional ${ }^{*, 1}$
}

\author{
The Contribution of the European Union to the Development of the \\ International Climate Change Regime: the EU Climate \& Energy \\ Package in the Context of the International Action
}

\author{
Dra. Rosa Giles Carnero \\ Profesora Titular de Derecho Internacional Público \\ de la Universidad de Huelva \\ giles@uhu.es
}

doi: http://dx.doi.org/10.18543/ced-57-2017pp193-215

\begin{abstract}
Sumario: I. Introducción.-II. El paquete europeo sobre clima y energía en el contexto del segundo periodo de cumplimiento del Protocolo de Kioto.-III. El paquete europeo sobre clima y energía como contribución determinada a nivel nacional en el contexto del Acuerdo de París.-IV. Conclusiones.
\end{abstract}

Resumen: La Unión Europea ha desplegado una estrategia de liderazgo en el régimen internacional en materia de cambio climático, basada en la credibilidad que le otorgaba el desarrollo de su política climática interna. La posición negociadora internacional se mostraba paralela al desarrollo de políticas europeas transversales que perseguían ambiciosos objetivos climáticos, al tiempo que se estructuraba una economía hipocarbónica que pretendía no perder competitividad. En este contexto, el paquete europeo sobre cambio climático y energía ha supuesto la principal contribución de la Unión Europea al desarrollo del régimen internacional sobre cambio climático, de forma que dotaba de contenido a los compromisos europeos diseñados en el segundo periodo de cumplimiento del Protocolo de Kioto, y en el Acuerdo de París; y servía de modelo para el diseño de políticas nacionales con significativos objetivos de mitigación del cambio climático.

* Recibido el 30 de noviembre de 2016 y aceptado el 29 de abril de 2017.

${ }^{1}$ El presente estudio se enmarca dentro del proyecto de investigación: «Las Respuestas del Derecho Internacional y Europeo a los Nuevos Riesgos y Amenazas Contra la Seguridad Humana» (RASEGUR), Plan Nacional de I+D+I (Ref.: DER2015-65906-P) y de la Red de Excelencia sobre «Los actuales desafíos del Derecho Internacional», del Plan Estatal de Investigación Científica y Técnica y de Innovación 2013-2016 (DER15-69273-RED). 
Palabras clave: Cambio climático; liderazgo de la Unión Europea; política climática de la Unión Europea.

Abstract: The European Union has deployed a leadership strategy in the international regime on climate change, based on the credibility of the development of its internal climate policy. The negotiating position was parallel to the development of horizontal European policies that pursued ambitious climate objectives, while designed a low-carbon and competitive economy. In this context, the EU climate \& energy package has been the main contribution of the European Union to the development of the international regime on climate change, on the one hand, these rules have provided content to the European obligations of the second commitment period of the Kyoto Protocol, and the Paris Agreement; on the other, have served as a model for designing national policies with significant objectives of climate change mitigation.

Keywords: Climate Change; EU Leadership; EU Climate Policy.

\section{Introducción}

El cambio climático se configura como uno de los grandes desafíos para una sociedad internacional que tiene que enfrentar el anuncio de una amenaza de consecuencias potencialmente catastróficas, mientras persiste un cierto grado de incertidumbre respecto de su repercusión concreta en las diferentes partes del planeta ${ }^{2}$. Ante este reto global, la principal respuesta internacional institucionalizada ha sido el desarrollo de un complejo régimen jurídico en materia de cambio climático, cuya evolución muestra la dificultad que presenta la negociación climática para alcanzar resultados ambientales efectivos. La estructura de este régimen internacional parte de la Convención Marco de las Naciones Unidas sobre el Cambio Climático, instrumento que articula el armazón negociador que ha permitido mantener abierto un proceso de negociación continuado desde la década de los noventa ${ }^{3}$. Este texto se diseñó

${ }^{2}$ Los diferentes informes del Grupo Intergubernamental de Expertos sobre el Cambio Climático han permitido avanzar en el consenso en torno a la gravedad de la amenaza que supone el cambio climático, y la necesidad de actuación urgente para enfrentarlo. Siguiendo esta línea, el quinto informe se presentó en octubre de 2014, y contiene la proyección de la evolución climática global en función de las diversas actuaciones que pudieran adoptarse. Vid. Rajendra K. Pachauri y Leo A. Meyer, eds. Cambio climático 2014: Informe de síntesis. Contribución de los Grupos de trabajo I, II y III al Quinto Informe de Evaluación del Grupo Intergubernamental de Expertos sobre el Cambio Climático (Suiza: IPPC, 2015).

3 Convención marco de las Naciones Unidas sobre el cambio climático, hecha en Nueva York (Estados Unidos) el 9 de mayo de 1992, y con entrada en vigor el 21 de marzo de 1994. UN Treaty Series, I-30822. 
como un primer paso que debía continuarse con la negociación de acuerdos específicos que asegurasen la estabilización de la temperatura global del planeta, de forma que la evolución del régimen puede seguirse a través del arduo proceso que llevó a la adopción de dos nuevos textos en 1997 y en 2015.

El Protocolo de Kioto supuso el primer acuerdo internacional para la disminución de las emisiones de gases de efecto invernadero, aunque con el importante límite de incluir obligaciones de reducción únicamente para los Estados que se calificaban como desarrollados conforme al Anexo I de la Convención Marco ${ }^{4}$. También este tratado se diseñó con la idea de que el régimen internacional tenía que desarrollarse paso a paso, de forma que se incluyó un primer periodo de cumplimiento que finalizaría en 2012, y el mandato de negociar uno posterior que fuera de aplicación desde esta fecha. Pese a esta previsión y ante la falta de compromiso de los Estados Partes, se agotó el plazo para negociar un segundo periodo de cumplimiento que pudiera estar en vigor desde 2013, y se llegó a una situación de parálisis en la negociación internacional que fue superada a través de la articulación de una doble vía de dialogo: mientras que se mantendría el esfuerzo para implementar un segundo periodo de cumplimiento en el sistema del Protocolo de Kioto; se abriría un nuevo espacio de negociación para alcanzar un acuerdo internacional que pudiera ser de aplicación a partir de $2020^{5}$. Ambas líneas de negociación se encuadraron en una fase que fue calificada como transitoria, y que abarcaría el parámetro temporal de 2013 a 2020. Como resultado de esta doble estrategia de dialogo se adoptó la Enmienda de Doha que conforma un segundo periodo de cumplimiento del Protocolo de Kioto a implementar desde 2013 a 2020, y que supone el único instrumento con medidas previstas para este periodo pese a que permanece pendiente de su entrada en vigor ${ }^{6}$; y el Acuerdo de París, mediante el que se pretende articular un nuevo sistema de obligaciones climáticas a aplicar a partir de $2020^{7}$.

${ }^{4}$ Protocolo de Kioto a la Convención marco de las Naciones Unidas sobre el cambio climático, adoptado en Kioto (Japón) el 11 de diciembre de 1997, y con entrada en vigor el 16 de febrero de 2005. UN Treaty Series, A-30822.

${ }_{5}$ Para un mayor desarrollo del análisis de esta fase transitoria, vid. Rosa Giles Carnero, «La acción internacional y europea en materia de cambio climático: construyendo la nueva fase 2013-2020», en La negociación de emisiones GEI en los mercados de carbono. Régimen y regulación, dir. Isabel Rodríguez Martínez (Pamplona: Thomson Reuters Aranzadi, 2014), 39-62.

${ }^{6}$ Enmienda de Doha al Protocolo de Kioto, adoptada en Doha (Catar) el 8 de diciembre de 2012, y pendiente de su entrada en vigor. La entrada en vigor de la Enmienda de Doha debe realizarse conforme a los artículos 20 y 21 del Protocolo de Kioto, que requieren la aceptación de tres cuartos de las Partes (144 Partes). A 9 de noviembre de 2016, 73 Partes habían aceptado la enmienda.

7 Acuerdo de París, adoptado en París (Francia) el 12 de diciembre de 2015, y con entrada en vigor el 4 de noviembre de 2016. 
En las sucesivas fases del devenir del régimen internacional en materia de cambio climático, la Unión Europea ha pretendido desarrollar un relevante papel de liderazgo, aunque no siempre se han conseguido los resultados perseguidos. La estrategia negociadora de la Unión Europea ha considerado que su capacidad de liderazgo se basaba en la idea de la diplomacia con el ejemplo, de forma que la política climática europea no se situaba como mero cumplimiento de la obligación internacional, sino que pasaba a convertirse en una relevante contribución para el avance del régimen jurídico internacional y en la fuente de credibilidad de los argumentos euro$\operatorname{peos}^{8}$. Desde este punto de vista, la posición negociadora de la Unión Europea tenía que resultar coherente con una actuación interna que precedía al desarrollo normativo que se solicitaba en la negociación internacional, y al que podía servir de modelo. En consecuencia, la acción climática europea se ha dirigido, en la esfera interna, al desarrollo de una acción normativa trasversal que permitiera avanzar en ambiciosos objetivos de reducción de emisiones de gases de efecto invernadero; mientras que en la esfera internacional ha defendido la necesidad de alcanzar acuerdos multilaterales con un impacto efectivo en la mitigación del cambio climático. El desarrollo de las políticas climáticas y de energía europeas se convertían, de esta forma, no sólo en un argumento para generar confianza en la negociación internacional, sino también en un modelo que mostraba la posibilidad de reducir emisiones de gases de efecto invernadero sin paralizar el crecimiento económico y actuando en un sistema multinivel.

Partiendo de los presupuestos señalados, la Unión Europea defendió con empeño la pervivencia del Protocolo de Kioto y la adopción de un segundo periodo de cumplimiento, en el que la política climática europea aparecía como un modelo de implementación de la norma internacional. No obstante, esta estrategia negociadora no produjo los resultados deseados

${ }^{8}$ La noción de liderazgo mediante el ejemplo ha interesado ampliamente a la doctrina internacionalista, que ha señalado tanto su potencialidad, como sus límites para lograr una posición relevante en la negociación global. A modo de ejemplo del interés y la controversia suscitados, pueden verse las reflexiones aportadas en: K. Bäckstrand y O. Elgström, The EU's Role in Climate Change Negotiations: From Leader to «Leadiator», Journal of European Public Policy 20, n. ${ }^{\circ} 10$ (2013): 1369-86; Joyeete Gupta y Michael J., Grubb, eds., Climate Change and European Leadership. A Sustainable Role for Europe? (Dordrecht: Kluwer Academic Publishers, 2000); Sebastian Oberthür y Lisanne Groen, «The European Union and the Paris Agreement: leader, mediator, or bystander?», WIREs Clim Change (2016), doi: 10.1002/wcc.445; C.F. Parker y C. Karlsson, «Climate Change and the European Union's Leadership Moment: An Inconvenient Truth?», Journal of Common Market Studies 48, n. ${ }^{\circ} 4$ (2010): 923-943; Sergio Salinas Alcega, El cambio climático: entre cooperación y conflicto. Propuestas desde el Derecho Internacional (Pamplona: Thomson Reuters Aranzadi, 2014); RKW Wurzel y J. Connelly, eds. The European Union as a Leader in International Climate Change Politics (London: Routledge, 2011). 
en un contexto en el que actores tan relevantes en la negociación climática como Estados Unidos y China se posicionaron a favor de un nuevo acuerdo internacional. Aunque finalmente fue posible la adopción de un segundo periodo de cumplimiento del Protocolo de Kioto, la Unión Europea quedó casi en solitario como la principal valedora de la pervivencia del sistema de obligaciones climáticas que conllevaba. Este escenario mostraba con claridad que el liderazgo climático había quedado desplazado hacia el eje conformado por China y Estados Unidos, de forma que la posición europea había perdido relevancia como motor de avance del régimen en materia de cambio climático. En este contexto, la Unión Europea adoptó una nueva posición apostando por un tratado multilateral y desplegando una renovada acción diplomática que facilitara los compromisos que llevarían a la adopción del Acuerdo de París. Después de transformar el objetivo, la diplomacia basada en el ejemplo volvía a convertirse en un instrumento útil para fomentar la confianza de los principales actores de la negociación climática, lo que resultaba especialmente relevante en un contexto en el que múltiples Estados en vías de desarrollo se habían mostrado reacios a aceptar compromisos climáticos relevantes.

Tanto en el segundo periodo de cumplimiento del Protocolo de Kioto como en el Acuerdo de París, se incluyeron, finalmente, diversos compromisos de mitigación del cambio climático, en el primero circunscritos a la reducción de emisiones de efecto invernadero en los Estados desarrollados; mientras que en el segundo con una aproximación más amplia respecto de las medidas a adoptar, y más general respecto a los Estados destinatarios de la obligación. En ambos sistemas, la posición de la Unión Europea ha resultado especialmente relevante a la hora de dotar de contenido a la obligación climática internacional, ya que la acción climática europea, desarrollada a través del denominado paquete sobre clima y energía, supone la concreción última del compromiso climático asumido, al tiempo que un modelo de implementación mediante los sistemas nacionales. La forma en la que se ha diseñado el sistema de obligaciones tanto en el segundo periodo de cumplimiento del Protocolo de Kioto, como en el Acuerdo de París, convierten en buena medida al sistema europeo en uno de los principales elementos con relevancia ambiental de la norma internacional. De esta forma, se produce la concreción jurídica de la diplomacia europea que pretende liderar con el ejemplo, y se inserta la contribución europea como el principal contenido del desarrollo del régimen internacional en materia de cambio climático.

El objetivo del presente estudio es el análisis de la relación expuesta entre el paquete europeo sobre clima y energía, y los sistemas de compromisos climáticos diseñados en el segundo periodo de cumplimiento del Protocolo de Kioto y en el Acuerdo de París. Esta relación conlleva el elemento más relevante de la contribución de la Unión Europea al desarrollo del régimen 
jurídico internacional en materia de cambio climático, ya que las medidas de mitigación se han convertido en la proyección concreta y más destacada del compromiso climático europeo, así como en modelo de implementación. A esto se añade que la relación expuesta muestra la capacidad de liderazgo climático de la Unión Europea, pero también sus límites en una situación de importantes contradicciones internas y un espacio internacional en el que ha perdido relevancia ambiental, económica y política. Aparecen en el momento de redactar este texto nuevos retos e incertidumbres que pueden afectar a la capacidad de implementar medidas eficaces de mitigación del cambio climático, tanto en el sistema europeo como en el internacional, por lo que una revisión de la experiencia adquirida en los últimos años puede servir de elemento de reflexión respecto a las medidas a adoptar ante los nuevos desafíos. El propósito del régimen internacional en materia de cambio climático es que la temperatura global del planeta no supere el nivel que puede conllevar consecuencias catastróficas; el de la Unión Europea es mostrarse como el modelo de una economía post carbono que pueda mantener un adecuado nivel de competitividad en una economía global; y ambas aspiraciones pretenden concretarse a través de un paquete de medidas sobre clima y energía que presenta aspectos contradictorios en su evaluación.

Conforme al objetivo señalado, en las páginas siguientes se aborda en un primer apartado el análisis de la acción de la Unión Europea en el desarrollo del segundo periodo de cumplimiento del Protocolo de Kioto; mientras que el segundo apartado se dedica al examen de la contribución prevista determinada a nivel nacional presentada por la Unión Europea en el marco de la negociación del Acuerdo de París, y que marca el inicio de los compromisos asumidos en el ámbito de este segundo instrumento. En ambos apartados se analizará el paquete europeo sobre clima y energía como destacada contribución al desarrollo del régimen internacional en materia de cambio climático, configurando una relación entre los sistemas europeo e internacional de mutua permeabilidad y continuada interacción. Se concluye el estudio con unas reflexiones sobre la experiencia de liderazgo mediante el ejemplo desarrollada por la Unión Europea, y la capacidad de esta aproximación para que la contribución europea resulte relevante en la consecución de los objetivos ambientales del régimen internacional en materia de cambio climático.

\section{El paquete europeo sobre clima y energía en el contexto del segundo periodo de cumplimiento del Protocolo de Kioto}

La aprobación del Acuerdo de París en 2015 ha eclipsado el recuerdo de un Protocolo de Kioto que se valora como un instrumento ineficaz para enfrentar el reto ambiental que supone el cambio climático. El impacto de 
las medidas de mitigación que aportó el primer periodo de cumplimiento de este instrumento, estuvo muy lejos de ser las requeridas ante la gravedad del fenómeno ambiental al que respondía. La interpretación del principio de responsabilidades comunes pero diferenciadas incluida en aquel primer desarrollo del régimen climático se apunta como la principal causa del fracaso en la obtención de los objetivos previstos. El sistema de obligaciones de reducción de emisiones de gases de efecto invernadero operaba únicamente sobre los Estados desarrollados incluidos en el Anexo I de la Convención Marco, lo que motivó que Estados Unidos no aceptara finalmente el tratado, y que no fuera posible articular compromisos de mitigación de las emisiones de gases de efecto invernadero de las economías emergentes. Esta situación provocó que, con el transcurrir de los años, los principales emisores de gases de efecto invernadero quedaran fuera del sistema de reducción de emisiones del Protocolo de Kioto, por lo que su eficacia quedó intensamente comprometida. El modelo acordado en París abandonó esta aproximación, incluyendo un sistema general a la vez que flexible destinado a que todas las Partes asuman diversos compromisos de actuación ante el cambio climático. Ahora bien, esta evolución del régimen internacional en materia de cambio climático no hace menos útil la valoración de la experiencia desarrollada durante el primer periodo de cumplimiento del Protocolo de Kioto y de la negociación del segundo, en los cuales la posición europea resulta especialmente destacada. La nueva fase que se abre parte ya de esta experiencia previa, y buena parte de las lecciones aprendidas y los límites detectados conformarán la acción a implementar.

La Unión Europea desarrolló un destacado liderazgo durante el primer periodo de cumplimiento del Protocolo de Kioto, favorecido en buena medida por la decisión estadounidense de no ratificar el tratado, y mantenerse al margen del sistema desarrollado conforme a sus previsiones. Sin embargo, esta posición de liderazgo en la negociación internacional climática fue diluyéndose a medida que se hacía necesario alcanzar el acuerdo que permitiera adoptar nuevos compromisos de reducción de emisiones de gases de efecto invernadero, en un nuevo periodo de cumplimiento que debía comenzar en $2013^{9}$. En la Decimoquinta Conferencia de las Partes de la Convención Marco y la Quinta reunión de las Partes del Protocolo de Kioto, celebradas en Copenhague en 2009, quedó patente la pérdida de relevancia

${ }^{9}$ Conforme al texto adoptado en 1997, el Protocolo de Kioto se configuró con un primer periodo de cumplimiento que finalizaría el 31 de diciembre de 2012, de forma que el segundo periodo tenía que diseñarse en función de las necesidades ambientales, sociales y políticas, y mediante el procedimiento de enmienda señalado en los artículos 2.9, 20 y 21.7 del mismo texto. 
negociadora de una Unión Europea que había acudido con el objetivo de convertirse en el principal protagonista en el foro global de negociación ${ }^{10}$. No puede enunciarse una causa única de este declive en su posición negociadora, no cabe duda de que la conjunción de una presencia más activa de Estados Unidos en la Conferencia de las Partes, y los estragos de una crisis económica que necesitaban de una mayor atención interna, explica en buena medida esta situación. Lo cierto es que, sean prioritariamente unas causas u otras, la Unión Europea se fue quedando aislada en una vía de negociación en la que su objetivo prioritario era adoptar un segundo periodo de cumplimiento del Protocolo de Kioto.

La estrategia de la Unión Europea en Copenhague se había basado en acudir con un desarrollo legislativo previo y unilateral, que aportara credibilidad a su posición negociadora. Es decir, la posición europea partía de aprovechar la experiencia desarrollada en el primer periodo de cumplimiento y asumir compromisos propios, al margen de que se produjese también la aceptación por otras Partes en el Protocolo. La diplomacia basada en el ejemplo definió la posición europea, de forma que la aprobación del denominado paquete de medidas sobre clima y energía para 2020 supuso el principal argumento para tratar de crear la confianza necesaria con la que alcanzar nuevos compromisos internacionales de reducción de emisiones de gases de efecto invernadero. El Consejo Europeo de marzo de 2007 configuró las líneas básicas de este paquete legislativo, que mediante una actuación transversal en la normativa europea pretende alcanzar, para 2020, el objetivo de reducir en un $20 \%$ las emisiones de gases de efecto invernadero, tomando como base el año 1990; incrementar la presencia de energías renovables en un $20 \%$; y mejorar la eficiencia energética en otro $20 \%{ }^{11}$.

Entre las medidas incluidas en este paquete legislativo, destacó la modificación del régimen de comercio de derechos de emisión, principal instrumento para reducir las emisiones de gases de efecto invernadero en el sistema europeo. No cabe duda de que el mercado de emisiones europeo supone un relevante modelo de esta técnica de mercado para la mitigación de emisiones de gases de efecto invernadero, cuyos logros y defectos suponen una experiencia observada por otras Partes en el régimen climático in-

10 Vid. Ana Barreira López, «El papel de la UE en las negociaciones de cambio climático», Cuadernos Europeos de Deusto, n. ${ }^{\circ} 42$ (2010): 41-57; y Rosa Giles Carnero, «La reunión de Copenhague de 2009: las nuevas negociaciones en torno al Principio de Responsabilidades Comunes pero diferenciadas de los Estados en materia de clima», Cuadernos Europeos de Deusto, n. ${ }^{\circ} 42$ (2010): 125-153.

11 Vid. Conclusiones del Consejo Europeo de 8 y 9 de marzo de 2007 [7224/1/07]. Estos objetivos fueron incluidos en la Comunicación de la Comisión, de 3 de marzo de 2010, EUROPA 2020: Una estrategia para un crecimiento inteligente, sostenible e integrador [COM (2010) 2020], que recoge la estrategia de crecimiento de la Unión Europea hasta 2020. 
ternacional ${ }^{12}$. El nuevo objetivo de este instrumento definido por el Consejo Europeo para presentarlo en la reunión de Copenhague fue el de reducir un $21 \%$ las emisiones de los sectores incluidos en el régimen respecto de las registradas en 2005, lo que suponía un relevante esfuerzo de mitigación, y abría una nueva fase en el desarrollo de la actividad en este mercado ${ }^{13}$.

La actuación sobre los sectores no controlados por el comercio de derechos de emisión se llevaría a cabo mediante el sistema de distribución del esfuerzo entre los Estados miembros, de forma que se realizó una atribución de porcentajes que incluían un amplio espacio entre la reducción del $20 \%$ atribuida a los países más ricos, y el incremento del $20 \%$ en los de peor situación ${ }^{14}$. Estas medidas quedarían complementadas con la actuación en el ámbito de la energía, en el que destacaban tanto las medidas legislativas en materia de energías renovables ${ }^{15}$; como las relativas a

12 La Directiva 2003/87/CE del Parlamento Europeo y del Consejo de 13 de octubre de 2003 por la que se establece un régimen para el comercio de derechos de emisión de gases de efecto invernadero en la Comunidad y por la que se modifica la Directiva 96/61/CE del Consejo (DOUE L 275 de 25 de octubre de 2003), estableció las bases de este mecanismo de reducción de emisiones a la atmósfera, y en su evolución se han incluido diferentes modificaciones que han pretendido superar algunos de los problemas que ha planteado su puesta en marcha. Sobre esta evolución vid. Pablo Antonio Fernández Sánchez, «El comercio de derechos de emisiones de $\mathrm{CO} 2$ en la UE, esperanza para el clima o nuevo modelo de mercadeo?», Revista de Derecho Comunitario Europeo, n. 39 (2011): 369-406; Beatriz Pérez de las Heras, «El régimen europeo de comercio de emisiones en el período post-Kiotoun mecanismo más armonizado y de responsabilidad diferenciada, paradigma de un mercado mundial de carbono», Revista General de Derecho Europeo, n. ${ }^{\circ} 26$ (2002): 1-26. Íñigo Sanz Rubiales y Susana Aníbarro Pérez, coord., Cambio climático y Unión Europea: presente y futuro del mercado europeo de emisiones (Valencia: Tirant lo Blanch, 2014).

${ }^{13}$ Las emisiones controladas por el Régimen de Comercio de Derechos de Emisión incluyen las procedentes del sector de la aviación y de las grandes instalaciones de los sectores eléctrico e industrial, y comprende, aproximadamente, el $45 \%$ de las emisiones de gases de efecto invernadero de la Unión Europea.

${ }^{14}$ Los sectores no incluidos en el Régimen de Comercio de Derechos de Emisión suponen el 55\% del total de las emisiones de la Unión Europea, y la distribución del compromiso en su reducción se realiza conforme a la Decisión n ${ }^{\circ}$ 406/2009/CE del Parlamento Europeo y del Consejo, de 23 de abril de 2009, sobre el esfuerzo de los Estados miembros para reducir sus emisiones de gases de efecto invernadero a fin de cumplir los compromisos adquiridos por la Comunidad hasta 2020 (DOUE L 140 de 5 de junio de 2009).

${ }_{15}$ La Directiva 2009/28/CE del Parlamento Europeo y del Consejo, de 23 de abril de 2009 , relativa al fomento del uso de energía procedente de fuentes renovables y por la que se modifican y se derogan las Directivas 2001/77/CE y 2003/30/CE (DOUE L 140 de 5 de junio de 2009) incluye el reparto entre los Estados miembros del esfuerzo para el incremento de las energías renovables; y sería completada por la Directiva (UE) 2015/1513 del Parlamento Europeo y del Consejo, de 9 de septiembre de 2015, por la que se modifican la Directiva 98/70/ CE, relativa a la calidad de la gasolina y el gasóleo, y la Directiva 2009/28/CE, relativa al fomento del uso de energía procedente de fuentes renovables (DOUE L 239 de 15 de septiembre de 2015). 
eficiencia energética ${ }^{16}$. Se conformaba, de esta forma, todo un paquete de medidas de carácter trasversal, con el objetivo de alcanzar un ambicioso reto de reducción de emisiones de gases de efecto invernadero y evolucionar hacia una economía hipocarbónica.

Como se ha señalado, la estrategia negociadora europea no produjo en Copenhague el resultado esperado, no sólo la Unión Europea perdió protagonismo en la negociación internacional, sino que los resultados obtenidos en el encuentro de 2009 distaron mucho de unas previsiones que lo anunciaban como la última oportunidad para evitar un vacío entre los sucesivos periodos de cumplimiento del Protocolo de Kioto. El acuerdo sobre un nuevo periodo de cumplimiento no fue posible hasta la octava sesión de la Reunión de las Partes en el Protocolo de Kioto, celebrada en Doha a finales de 2012, en la que fue adoptada una enmienda a este tratado que establece nuevos compromisos para un segundo periodo de cumplimiento comprendido entre 2013 a 2020. El acuerdo para la adopción de este nuevo texto se logró, pero la eficacia ambiental del segundo periodo de cumplimiento quedaba muy limitada debido al escaso número de Estados Partes incluidos en el Anexo I de la Convención Marco que se mostraron dispuestos a aceptar nuevos compromisos de reducción. Las Partes que son Estados desarrollados, y que asumirán obligaciones cuantificadas de reducción de emisiones de gases de efecto invernadero en el nuevo periodo, son menos que las que lo hicieron en el texto inicial de 1997, y suponen un escaso porcentaje de las emisiones globales ${ }^{17}$.

16 En materia de eficiencia energética también se contemplan compromisos de los Estados miembros para su incremento, vid. en particular: la Directiva 2012/27/UE del Parlamento Europeo y del Consejo, de 25 de octubre de 2012, relativa a la eficiencia energética, por la que se modifican las Directivas 2009/125/CE y 2010/30/UE, y por la que se derogan las Directivas 2004/8/CE y 2006/32/CE (DOUE L 315 de 14 de noviembre de 2012); y la Directiva 2010/31/UE del Parlamento Europeo y del Consejo, de 19 de mayo de 2010, relativa a la eficiencia energética de los edificios (DOUE L 153 de 18 de junio de 2010).

17 Al comparar el listado de los Estados con compromisos de reducción en el primer periodo de cumplimiento y los que los asumirán en el segundo, aparecen diversas situaciones. Canadá ha sido el único Estado que ha abandonado el Protocolo de Kioto conforme al procedimiento de denuncia de su artículo 27. Los Estados que asumen nuevos compromisos de reducción de emisiones para el periodo 2013-2020 son Australia, Croacia, Liechtenstein, Mónaco, Noruega, Suiza y Ucrania, a los que se suma la Unión Europea y sus Estados miembros. Los Estados desarrollados que no han aceptado nuevos compromisos cuantificados de reducción de emisiones siguen siendo Partes en el Protocolo de Kioto, y también entre ellos aparecen diferencias. Así, por ejemplo, mientras que Nueva Zelanda anunció que adoptaría medidas de reducción de emisiones para el periodo 2013-2020 con arreglo a los objetivos de la Convención Marco, la Federación Rusa y Japón comunicaron que no asumirían compromisos de reducción en el nuevo periodo. 
En lo que respecta a la Unión Europea, si se acude a la columna del Anexo B de la Enmienda en la que aparecen los porcentajes de reducción de emisiones comprometidos, y se hace una lectura conjunta con las declaraciones de las Partes incluidas en la misma Decisión de la Reunión de las Partes, puede afirmarse que el compromiso asumido es el de reducir en un $20 \%$ sus emisiones de gases de efecto invernadero tomando como año base general 1990, a lo que se suma el ofrecimiento de incrementar el porcentaje al 30\% para el año 2020 «a condición de que otros países desarrollados se comprometen a aplicar reducciones comparables de sus emisiones y los países en desarrollo hagan una contribución adecuada con arreglo a sus responsabilidades y capacidades respectivas ${ }^{18}$. En el enmendado Protocolo de Kioto la Unión Europea asumiría, por tanto, unos porcentajes de reducción en todo caso, que podrían incrementarse en función del éxito con el que fuera desarrollándose el proceso negociador en el marco de la Conferencia de las Partes.

Aunque se ha enunciado la obligación internacional que asumiría la Unión Europea, no puede olvidarse que tanto el compromiso de reducción del $20 \%$ como el eventual incremento al $30 \%$, suponen el objetivo general que ya había sido acordado de forma unilateral previamente a la reunión en Copenhague. Como ya se ha señalado, la Estrategia 2020, diseñada como la hoja de ruta del desarrollo económico de la Unión Europea en la presente década, asume estos objetivos como parte de un crecimiento que debe ser sostenible a largo plazo; mientras que el paquete sobre clima y energía supone un amplio entramado en el que tanto la política de cambio climático como la energética confluyen para alcanzar los objetivos previstos de reducción de emisiones de gases de efecto invernadero. La implementación europea del segundo periodo del enmendado Protocolo de Kioto supone, de hecho, el cumplimiento de su normativa previamente decidida, ya que el diseño mismo de este nuevo periodo se debe en buena medida a la actividad de la Unión Europea y supone la proyección internacional de los objetivos diseñados en la normativa interna ${ }^{19}$. De esta forma, las previsiones del segundo periodo de cumplimiento del Protocolo de Kioto y del paquete sobre clima y energía quedan interrelacionadas, y suponen el escenario de influencia internacional y el compromiso interno de la Unión Europea en materia de cambio climático.

18 Vid. Decisión 1/CMP.8, Enmienda al Protocolo de Kioto de conformidad con su artículo 3, párrafo 9 (enmienda de Doha).

19 Ver en este sentido las Conclusiones del Consejo de 25 de octubre de 2012, para la preparación de la COP18-CMP8, en las que puede observarse la interacción entre ambos sistemas. 
La escasa participación en el sistema de obligaciones definidas sitúa, además, a la Unión Europea como el principal escenario en el que se desplegarán los efectos ambientales del Protocolo de Kioto. Si se repasa el listado de Estados con compromisos de reducción en el periodo de 2013 a 2020, aparece que, a excepción de Australia, el territorio europeo es el ámbito en el que este instrumento desplegará su limitada eficacia. Puede afirmarse, por tanto, que el cumplimiento de los objetivos del Protocolo de Kioto va a coincidir, en buena medida, con el cumplimiento de los objetivos de la política climática de la Unión Europea. De nuevo, esta situación sitúa al modelo de actuación climática de la Unión Europea como el principal escenario en el que se ha desarrollado una experiencia que resultará valiosa a la hora de afrontar nuevas etapas en el régimen climático. El modelo europeo de reducción de emisiones aparece en el contexto del segundo periodo de cumplimiento del Protocolo de Kioto como la principal contribución a esta etapa del régimen internacional, no sólo como la implementación de unos compromisos concretos, sino como un espacio de experiencia en la aplicación de medidas de mercado y de actuación multinivel.

La incidencia ambiental de la Enmienda de Doha será, en cualquier caso, pequeña debido al escaso número de Estados del Anexo I que han aceptado nuevos compromisos de reducción, pero a esto debe añadirse que resulta incierta la posibilidad de que finalmente se alcance el número requerido de aceptaciones para su definitiva entrada en vigor. Pese a su escaso impacto ambiental, la participación en este segundo periodo de cumplimiento supondría una muestra del compromiso adquirido en el desarrollo de medidas de mitigación del cambio climático, así como la continuidad de la experiencia de actuación institucional que se había desarrollado en el primer periodo. Debido a esto, la Unión Europea ha defendido la conveniencia de su participación, e incluso ha anunciado la fecha de su aceptación en diversas ocasiones, pese a todo lo cual, no ha sido posible la prestación del consentimiento. Algunos Estados miembros han aceptado la enmienda, como por ejemplo Rumanía, el 3 de mayo de 2016, e Italia el 18 de julio de 2016, ésta segunda introduciendo una declaración sobre la competencia de la $\mathrm{UE}^{20}$. La adopción

20 En particular, Italia ha declarado al aceptar la enmienda que: «with regard to the instrument of acceptance of the Doha Amendment to the Kyoto Protocol deposited on 18 July 2016, the Government of Italy would like to point out that given the nature of the rights and obligations provided for therein and taking into account the legal system of the European Union (the EU) and its Member States, implementation will only be possible and obligations will come into effect once the EU and all its Member States will have deposited their relevant instruments of acceptance». Puede consultarse el estado de aceptación y las declaraciones presentadas en el sitio web: https://treaties.un.org/Pages/ViewDetails .aspx?src=TREATY\&mtdsg_no=XXVII-7-c\&chapter=27\&clang=_en 
en el verano de 2015 de una Decisión del Consejo respecto a la aceptación de la enmienda pareció anunciar que se produciría antes de la celebración del encuentro en París previsto para el mismo año, finalmente no fue posible, y este último gesto no se ha llegado a producir ${ }^{21}$.

Aunque el efecto práctico de la ratificación europea es escaso, debido a que la Unión Europea ha asumido los objetivos del segundo periodo de cumplimiento de forma unilateral mediante el paquete sobre clima y energía para 2020, la aceptación de la enmienda tiene una importante trascendencia política en un actor que pretende desplegar una estrategia basada en el ejemplo. Aunque la obligación ambiental no aceptada se está cumpliendo conforme a la normativa europea en vigor, la aceptación continúa teniendo importancia tanto para mostrar el compromiso con el sistema internacional institucionalizado para la mitigación y adaptación al cambio climático, como respecto a la participación en un instrumento que incluye concretas obligaciones de información y un sistema institucionalizado de cumplimiento. Aunque el modelo de reparto de compromisos climáticos del Protocolo de Kioto ha sido abandonado al adoptar el Acuerdo de París, la experiencia acumulada en los años de su vigencia será aprovechada en las nuevas fases del régimen. En este aspecto, la contribución de la Unión Europea ha sido relevante a la hora de convertir al modelo europeo en el principal referente para la aplicación de medidas de mitigación, pero los problemas para poder finalizar una aceptación a la Enmienda de Doha resultan preocupantes a la hora de valorar la capacidad de mantener el compromiso con la actuación climática en los próximos años.

\section{EI paquete europeo sobre clima y energía como contribución determinada a nivel nacional en el contexto del Acuerdo de París}

Como ya se ha apuntado, el Protocolo de Kioto se caracterizó por utilizar una interpretación del principio de responsabilidades comunes pero diferenciadas de los Estados, que se limitaba a hacer recaer los compromisos de mitigación sólo sobre los desarrollados incluidos en el Anexo I de la Convención Marco. El sistema adoptado en 1997 conjugó la aplicación del concepto de equidad, conectado a las emisiones históricas, y del principio del derecho al desarrollo, para promover un sistema en el que Estados con un alto potencial emisor de gases de efecto invernadero no

${ }^{21}$ Vid. Council Decision on the conclusion, on behalf of the European Union, of the Doha Amendment to the Kyoto Protocol to the United Nations Framework Convention on Climate Change and the joint fulfilment of commitments thereunder, 12 de junio de 2015 , [10400/5/14 REV 5]. 
asumirían compromiso alguno de limitación por ser calificados de países en vías de desarrollo. Esta situación conllevó una importante ineficacia para la consecución de los objetivos ambientales, y se fue agravando a medida que se iba produciendo un cambio de circunstancias que convertían a algunos Estados en vías de desarrollo en los principales emisores de gases de efecto invernadero, y les dotaba de mayor protagonismo económico y comercial.

Esta experiencia del Protocolo de Kioto mostró la necesidad de buscar una nueva aproximación para el desarrollo del régimen climático, y en la decimoséptima sesión de la Conferencia de las Partes, celebrada en Durban a finales de 2011, se acordó el inicio de un proceso para «elaborar un protocolo, otro instrumento jurídico o una conclusión acordada con fuerza legal» en el contexto de la Convención Marco, y que debía culminar con la adopción de un nuevo acuerdo internacional en la vigésima primera sesión de la Conferencia de las Partes, a celebrar en París en 2015 22 . El que posteriormente se calificaría de Acuerdo de París tenía que ser un nuevo acuerdo climático global y ambicioso, que permitiera lograr el objetivo de evitar la subida de la temperatura global del planeta por encima de los $2 .{ }^{\circ} \mathrm{C}$, y supusiera el marco jurídico de referencia único a partir de 2020.

El concepto de las contribuciones previstas determinadas a nivel nacional (INCDs, por sus siglas en inglés) apareció en el proceso negociador del nuevo texto como la solución para incluir compromisos generales para las Partes en la Convención Marco que, a su vez, tuvieran en cuenta las diferentes circunstancias nacionales. La Conferencia de las Partes, en su décimo noveno periodo de sesiones, celebrado en Varsovia en noviembre de 2013, invitó a las Partes a iniciar o desarrollar la preparación de sus INDCs como nuevo método para asegurar el avance del régimen internacional en materia de clima, llamamiento que fue reiterado en su vigésima reunión celebrada en Lima en diciembre de $2014^{23}$. Se estableció así un procedimiento en el que se animaba a las Partes a presentar su previsión respecto a sus planes nacionales de actuación en materia de cambio climático a partir de 2020, de forma que estas aportaciones se convirtieran en la base de la negociación de los compromisos internacionales que estarían dispuestas a asumir. En este nuevo modelo, el diseño de la norma internacional parte de una primera actuación de los sujetos que habrán de prestar el consentimiento y proceder a

22 Vid. Decisión 1/CP.17, Establecimiento de un Grupo de Trabajo Especial sobre la Plataforma de Durban para una Acción Reforzada, par. 2 y 4.

${ }^{23}$ Vid. la Decisión 1/CP.19, Intensificación de los trabajos relativos a la Plataforma de Durban, 19..$^{\circ}$ periodo de sesiones, celebrado en Varsovia del 11 al 23 de noviembre de 2013, para. 2; y Decisión 1/CP.20, Llamado de Lima para la Acción Climática, 20 ${ }^{\circ}$ periodo de sesiones, celebrado en Lima del 1 al 14 de diciembre de 2014, para. 9. 
su aplicación, al tiempo que diferencia los compromisos en función de la situación específica de cada uno de ellos. En la búsqueda de la efectividad negociadora, este procedimiento pretendía asegurar el compromiso efectivo con las medidas internacionales que pudieran adoptarse, y para ello se aceptó asumir un sistema flexible de obligaciones en el que incluyeran diferentes tipos de actuaciones que compartiesen un efecto final de beneficio climático.

La figura de las INDCs sería trasladada al Acuerdo de París que las incluyó como la pieza clave del sistema de obligaciones climáticas que instituye. En su artículo 4, el tratado recoge la obligación de las Partes de preparar, comunicar y aplicar las contribuciones determinadas a nivel nacional (NDCs, en sus siglas en inglés) que permitan estabilizar la temperatura del planeta a niveles seguros. Cada NDCs tendrá que revisarse cada cinco años, incrementando su ambición respecto a la consecución de los objetivos climáticos previstos. De esta forma, se establece un sistema progresivo en el que todas las Partes asumen compromisos independientemente de si son desarrolladas o no, pero con una extensa diferenciación en función de las circunstancias específicas de cada una. Desde una óptica global, se inicia un proceso de «descarbonización» general a través de la actuación diferenciada en cada uno de los sistemas nacionales.

Respecto al contenido que deberá incluirse en las NDCs, el artículo 4 va a remitir a la experiencia desarrollada en la negoción del Acuerdo de París. Durante las sucesivas reuniones de la Conferencia de las Partes fueron incluyéndose diferentes referencias que ayudaron a conformar el contenido mínimo que tendrían que incluir. En su décima novena reunión, la Conferencia de las Partes señaló que las INDCs debían incluir una información que facilitara la claridad, la transparencia, y la comprensión de las medidas a adoptar ${ }^{24}$; mientras que en la siguiente reunión se precisó algo más el contenido al señalar que «deberá representar un avance con respecto a los compromisos actuales de esa Parte» y «podrá incluir, entre otras cosas, según corresponda, información cuantificable sobre el punto de referencia (con indicación de un año de base, cuando proceda), los plazos y/o períodos de aplicación, el ámbito y la cobertura, los procesos de planificación, los supuestos y los enfoques metodológicos, incluidos los destinados a estimar y contabilizar las emisiones y, cuando sea el caso, las absorciones antropógenas de gases de efecto invernadero, y una indicación de los motivos por los que consideren que su contribución prevista determinada a nivel nacional es justa y ambiciosa, a la luz de sus respectivas circunstancias nacionales, y de la manera en que contribuye a la consecución del objetivo de la

${ }^{24}$ Vid. Decisión 1/CP.19, para. 2 (b) y (c). 
Convención» ${ }^{25}$. A esto se añade que también en esta última reunión se invitó a todas las Partes a aportar sus planes de adaptación o a incluir un componente de adaptación en su contribución, de forma que se aceptaba que en la INDCs se incluyeran también compromisos e información sobre este apartado ${ }^{26}$.

El procedimiento de presentación de las INCDs resultó un éxito a juzgar por las contribuciones presentadas, que supusieron el punto de partida para crear un clima de confianza mediante el que se adoptó el Acuerdo de París, y pudo obtener las ratificaciones necesarias para su entrada en vigor en escasos once meses. Durante este proceso, la Unión Europea y sus Estados miembros se declararon comprometidos con el objetivo de adoptar un acuerdo global con capacidad jurídica vinculante. La Unión Europea reestructuró, de esta forma, su estrategia de negociación para tratar de mantener su capacidad protagónica en un escenario climático en el que un nuevo orden internacional se definía, tanto en los objetivos a alcanzar como en lo actores relevantes. En las Conclusiones del Consejo Europeo, celebrado en octubre de 2014, se declaró el compromiso para alcanzar este acuerdo internacional, al tiempo que se avanzó en las medidas internas que se desarrollarían en su contexto ${ }^{27}$. En este sentido, el Consejo Europeo concluyó con la decisión de apoyar una acción eficaz en materia de cambio climático a nivel internacional, al tiempo que con la de avanzar en el objetivo interno de configurar una economía baja en carbono. Una vez más se trataba de mostrar que la posición negociadora internacional resultaba coherente con un desarrollo legislativo interno, que se podía anticipar a la conformación definitiva del compromiso internacional, y servir de referente al resto de Partes negociadoras.

En aquella reunión, el Consejo europeo asumió que los objetivos previstos para 2020 se cumplirán, por lo que era posible avanzar en otros más ambiciosos y retomar una senda que se había desdibujado en las reuniones dedicadas a los urgentes problemas de la crisis económica. En consecuencia, la Unión Europea anunció su compromiso de reducir para 2030 un $40 \%$ las emisiones sobre el año base de 1990, así como un incremento al $27 \%$ de la presencia de renovables en el sistema energético y el mismo incremento en eficiencia energética. Se señaló, además, que el comercio europeo de derechos de emisión debía continuar como el principal instrumento para conseguir el objetivo de reducción de emisiones, a lo que se sumaría el paquete de medidas que fueran necesarias en el marco de la cada una de las políticas

25 Vid. Decisión 1/CP.20, para. 10 y 14.

26 Ver Decisión 1/CP.20, para. 12.

27 Conclusiones del Consejo Europeo de 23 y 24 de octubre de 2014, EUCO 169/14, 24 de octubre de 2014 . 
sectoriales. Es decir, el Consejo dibujaba una nueva fase del paquete europeo sobre clima y energía que transcurrirá en el periodo de 2020 a 2030, aceptando la continuidad de las principales líneas de actuación desarrolladas que reciben un nuevo impulso y revisión. El paquete de medidas continuaba y progresaba, y en las conclusiones se subrayaba la idea del esfuerzo añadido, cuestión que sería clave para trasladarla a un proceso de negociación en el que se pretendía que la acción internacional pudiera avanzar sobre la base de unos compromisos cada vez más ambiciosos a desarrollar en las políticas nacionales.

Con esta determinación la Unión Europea muestra que la cuestión del cambio climático sigue siendo prioritaria en el desarrollo de su política internacional, y que su estrategia para mantener su influencia en los foros de negociación sigue siendo convertirse en el modelo de una sociedad baja en carbono. La Unión Europea se situaba, de esta forma, en la avanzadilla de los Estados dispuestos a colaborar con el régimen internacional, creando una confianza mutua en la que desarrollar el consenso sobre acciones ambientales cada vez más ambiciosas. La nueva fase del paquete sobre clima y energía pasaría, una vez más, a convertirse en el contenido del compromiso climático internacional que desde el ámbito europeo se ofrecía al régimen internacional, y en esta ocasión quedaría recogido en la INDCs. Si el segundo periodo de cumplimiento del Protocolo de Kioto había mostrado una situación en la que la política climática interna de la Unión Europea se había convertido en el principal contenido; en esta nueva fase esa misma política quedaría recogida como una de esas contribuciones nacionales con las que el régimen internacional pretendía cumplir sus objetivos. Finalmente, la INDC europea fue presentada de forma conjunta por Latvia, que ostentaba la presidencia del Consejo Europeo, y por la Comisión Europea en representación de la Unión Europea y sus Estados Miembros el 6 de marzo de $2015^{28}$.

Conforme al texto presentado, la Unión Europea y sus Estados miembros actuarán conjuntamente para la implementación de la contribución nacional a los objetivos ambientales del régimen internacional en materia de cambio climático que se describe. En particular, se señaló el compromiso de reducir en, al menos, un $40 \%$ las emisiones de gases de efecto invernadero tomando como base 1990, y durante el periodo comprendido entre el 1 de enero de 2021 y el 31 de diciembre de 2030. Como puede observarse, este objetivo se corresponde con el anunciado en el Consejo Europeo de octubre de 2014, y se conecta con la nueva fase del paquete europeo so-

${ }^{28}$ La Secretaría de la Convención Marco ha articulado un registro público on line en el que pueden consultarse las diferentes contribuciones nacionales, vid. http://www4.unfccc.int/ submissions/indc/Submission\%20Pages/submissions.aspx. 
bre clima y energía. Deben destacarse además dos previsiones, la primera es que la reducción de las emisiones pretende ser únicamente doméstica, ya que se informa del compromiso de no utilizar mecanismos de mercado; la segunda se refiere al hecho de que la acción presentada se circunscribe únicamente al ámbito de la mitigación, sin que se haya incluido ninguna previsión respecto a adaptación.

En el texto de la contribución se señaló que la implementación de la INDC se realizará mediante el despliegue de las medidas incluidas en el paquete europeo sobre clima y energía. Se recordó las actuaciones ya en marcha y cuyo periodo de vigencia es hasta 2020, así como el hecho de que se insertan en el sistema del segundo periodo de cumplimiento del Protocolo de Kioto, y por tanto en un sistema de verificación internacional, de forma que quedaba patente la línea de continuidad de los compromisos climáticos europeos. El esfuerzo agregado en esta nueva fase se concretaba en el anuncio de nuevas medidas que empezarían a tramitarse en 2015 y 2016, dirigidas a alcanzar los nuevos objetivos para 2030, y que afectarían tanto a los sectores incluidos en el mercado europeo de emisiones, como al resto. El enfoque trasversal, coherente con el principio de integridad del artículo 11 del Tratado de Funcionamiento de la Unión Europea, se mantiene vigente en esta nueva fase del paquete sobre clima y energía, de forma que se señala expresamente la actuación en los ámbitos de energía, procesos industriales y uso de productos, agricultura, residuos, el uso de la tierra, el cambio del uso de la tierra y la silvicultura ${ }^{29}$.

La INDC europea se insertó, por tanto, en un proceso que ha permitido la adopción y rápida entrada en vigor del Acuerdo de París, y se configuró como la contribución europea al esfuerzo por avanzar en el régimen climático internacional ${ }^{30}$. No obstante, nuevos interrogantes se abren respecto a las fases subsiguientes. La primera cuestión es la de la ratificación del Acuerdo de París que, como tratado mixto, debe realizarse conforme al artículo 218 del Tratado de Funcionamiento de la Unión Europea. La Unión Europea presentó su aceptación el 5 de octubre de 2016, como también lo hicieron algunos Estados miembros como Alemania, Austria, Francia, Hungría, Malta o Portugal. Por muy diversas razones, otros Estados miembros

29 En particular, se incluye la referencia a la Decisión 529/2013/UE del Parlamento Europeo y del Consejo, de 21 de mayo de 2013, sobre normas contables aplicables a las emisiones y absorciones de gases de efecto invernadero resultantes de actividades relativas al uso de la tierra, el cambio de uso de la tierra y la silvicultura y sobre la información relativa a las acciones relacionadas con dichas actividades (DOUE L 165 de 18 de junio de 2013).

${ }^{30}$ La comparación de la INDC europea con la de diferentes Estados desarrollados muestra una mayor ambición de los objetivos ambientales previstos. 
han ido retrasando su aceptación, de forma que aún quedan algunos por prestar su consentimiento al nuevo tratado. Completado el proceso de ratificaciones europeas, quedaría el de tramitar las reformas requeridas del paquete sobre clima y energía.

La nueva fase legislativa se enmarcaría en las previsiones dela Comisión Europea contenidas en la Hoja de Ruta de 2050 hacia una economía sin carbono, y requiere la revisión normativa aún en fase de discusión en diversos sectores como el mercado de emisiones, el sistema de distribución del esfuerzo, y la estrategia de transportes; o ya en fase de propuestas como en los sectores de las energías renovables, la eficiencia energética y los mercados de la energía ${ }^{31}$. En este escenario las divergencias y los recientes problemas surgidos en el proceso de integración europea tendrán que enfrentarse para llegar a nuevos acuerdos. La ratificación progresiva del Acuerdo de París, parece mostrar un dato positivo respecto de la implicación de los Estados miembros en la consecución de unos objetivos climáticos que se han imbricado en la base de un determinado modelo de desarrollo económico y social ${ }^{32}$. No obstante, la tensión surgirá sin duda entre los aquellos socios más proclives al avance de la regulación europea hacia objetivos más ambiciosos, y los más reticentes ${ }^{33}$.

\section{Conclusiones}

El cambio climático supone uno de los desafíos más trascendentes para la sociedad internacional contemporánea, si no se actúa a tiempo las

31 Vid. Comunicación de la Comisión, de 8 de marzo de 2011, Hoja de ruta hacia una economía hipocarbónica competitiva en 2050 [COM(2011) 112].

32 El modelo de crecimiento europeo supone la opción de una estrategia económica que permita avanzar en la consecución de objetivos climáticos, de forma que transciende lo ambiental para convertirse en una cuestión de toma de decisiones sobre la naturaleza del desarrollo europeo. Desde esta perspectiva y a modo de ejemplo, deben tenerse en cuenta reflexiones como las de Sebastian Oberthür, que entre los diferentes aspectos de la política europea a revisar para mantener el liderazgo en la escena internacional, incluye la conveniencia de facilitar e incentivar la acción de instituciones regionales y locales, y de actores privados. Vid. Sebastian Oberthür, «The European Union in Crisis: What Future for the EU in International Climate Policy?», IES Policy Brief 18 (2016): 1-4.

${ }^{33}$ Los objetivos climáticos pretendidos van a requerir actuaciones ambiciosas en diversos sectores que pueden poner de manifiesto las reticencias que algunos Estados ya han mostrado en el desarrollo de las medidas actualmente en vigor. El caso de España en materia de renovables, puede resultar ilustrativo de las tensiones ya producidas y que tendrán que enfrentarse en el proceso de modificación legislativa requerida. Vid. Manuela Mora Ruíz, «La ordenación jurídico-administrativa de las energías renovables como pieza clave en la lucha contra el cambio climático: ¿un sector en crisis?», Actualidad Jurídica Ambiental, n. ${ }^{\circ} 60$ (2016): 82-84. 
consecuencias pueden ser catastróficas para la vida humana en el planeta, pero la actuación efectiva conlleva un importante cambio de vida y del modelo de desarrollo occidental. La llamada a la actuación comporta replantear cómo vivir en un mundo limitado de recursos, y con un equilibrio ecológico sensible, el reto es evolucionar hacia sociedades hipocarbónicas en las que los sistemas energéticos y de consumo sean esencialmente diferentes a los desplegados en las últimas décadas en los países desarrollados. Ante este reto global, la Unión Europea tiene una cualificada responsabilidad, por incluir un espacio con importantes emisiones históricas que colaboraron a general el problema; pero también una interesante oportunidad de convertirse en modelo de una sociedad cuyo crecimiento económico y social se realice acompañado de una disminución de sus emisiones de gases de efecto invernadero. La aproximación que la Unión Europea ha elegido ante el cambio climático no tiene únicamente consecuencias ambientales, sino que incide en el modelo económico y social a desarrollar en el espacio europeo. Esta idea ha quedado clara en la Estrategia 2020, de forma que la Unión Europea ha optado por un determinado tipo de crecimiento económico en el que resulta imprescindible el desarrollo de medidas como las incluidas en el paquete sobre clima y energía y que se han descrito en el presente trabajo.

Si en la esfera interna la apuesta de la Unión Europea por el cumplimiento de objetivos climáticos se interconecta con un concepto de desarrollo que pretende ser a un tiempo sostenible y competitivo, en la esfera internacional ha mantenido su esfuerzo por ostentar el liderazgo en el desarrollo del régimen internacional en materia de cambio climático. El devenir de la negociación climática global situó primero a la Unión Europea en una posición ventajosa, ante la ausencia de Estados Unidos en el sistema del Protocolo de Kioto, pero después le restó relevancia ante el retorno de aquel a la escena climática y el cambio de circunstancias económicas, sociales y ambientales que situaban a las economías emergentes, y especialmente a China, en la primera línea de relevancia. La Unión Europea tuvo que reestructurar sus objetivos para mantenerse entre los principales actores negociadores del nuevo tratado internacional que se materializaría en el Acuerdo de París, pero se mantuvo la misma estrategia durante toda la evolución del régimen, basada en conectar su posición internacional con la acción interna desarrollada de forma unilateral. Esta posición dotaba de credibilidad a la postura negociadora europea, y le ha permitido establecer diversas alianzas en un escenario en el que ha resultado difícil desarrollar una posición relevante más allá del eje conformado por Estados Unidos y China.

En el contexto descrito, el paquete europeo sobre cambio climático y energía se ha convertido, al mismo tiempo, en el desarrollo de una política europea con unos objetivos ambientales, sociales y económicos estableci- 
dos; en el contenido del compromiso climático internacional asumido por la Unión Europea; y en un modelo de implementación de la norma climática internacional que asumía objetivos ambiciosos y podía servir de ejemplo para otros Estados Partes. Debido a la posición adoptada por la Unión Europea y a las características del régimen internacional en materia de cambio climático, el paquete sobre clima y energía es el núcleo de una interacción entre la normativa europea y la internacional que va más allá de las cuestiones clásicas de la recepción y cumplimiento de la norma internacional, y transforman a la legislación europea en la más relevante contribución a la gestión internacional del cambio climático. La normativa europea se convierte en el escenario en el que experimentar acciones nacionales de mitigación que puedan mostrarse eficaces, y esta experiencia es lo que se traslada al sistema internacional previamente convertido en obligación interna. Se produce, de esta forma, un dialogo entre sistemas el que la experiencia de gestión se estructura en un sistema multinivel.

La negociación basada en el ejemplo alcanza, además, pleno significado en el sistema establecido por el Acuerdo de París. El fundamento de este nuevo texto es precisamente el dialogo y la interacción continuada entre el régimen internacional y los sistemas nacionales, de forma que sea la actuación de las Partes la que concrete un compromiso internacional que no está preestablecido, y que se mantiene abierto a su mejora en periodos de cinco años. La actuación de la diplomacia europea, y en particular de la francesa al situarse como anfitriona, influyó en el resultado de una nueva reunión de la Conferencia de las Partes en la Convención Marco que pudiera dar un resultado concluyente. Esta diplomacia partía de una estrategia en la que resultaba familiar que el avance de la norma internacional pudiera apoyarse en el avance previo de las Partes que pudieran liderar el proceso, y unido al nuevo dialogo entre Estados Unidos y China, se convirtió en la base sobre la que diseñar una nueva etapa de la acción internacional. En esta nueva fase, el paquete europeo en materia de clima y energía se sitúa de nuevo como el contenido básico de lo que será el compromiso adquirido a nivel internacional, pero además resulta un modelo de actuación con instrumentos como el mercado de emisiones que pueden presentar ya resultados de aplicación, y por lo tanto, la posibilidad de interaccionar a su vez con sistemas nacionales de aplicación.

Nuevas incertidumbres se presentan tanto respecto del futuro del régimen internacional en materia de cambio climático, como de la reforma requerida del paquete europeo sobre clima y energía para alcanzar los objetivos de 2030. En un escenario u otro, el cuestionamiento es el del compromiso con objetivos ambiciosos de mitigación del cambio climático, y con la consecuente evolución hacía el desarrollo hipocarbónico. Los obstáculos aparecen desde diversos frentes, mostrando una vez más la difi- 
cultad de los Estados para asumir el compromiso de una acción ambiental costosa en el corto plazo. Los diferentes problemas internos de la Unión Europea podrán reflejarse en la reforma de la normativa europea en fase de preparación, y esas dificultades mostrarán también si mantiene la capacidad de liderazgo en una negociación internacional que puede tomar un nuevo y difícil rumbo con el cambio de la presidencia estadounidense. Ante los retos, las contribuciones nacionales siguen presentándose en el marco del Acuerdo de París, por lo que a la Unión Europea se le ofrece un nuevo y propicio escenario para continuar desarrollando una relevante contribución al avance de la acción internacional en materia de cambio climático.

\section{Sobre el autor}

Rosa Giles Carnero es Profesora Titular de Derecho Internacional Público y Relaciones Internacionales de la Universidad de Huelva (España).

Ha sido coordinadora del Módulo Jean Monnet La Política de la Unión Europea en materia de cambio climático (2011-2014), centrando sus líneas de investigación en el marco del Derecho Europeo, en las políticas europeas ambiental y climática, en la acción europea para la igualdad de género, y en seguridad medioambiental. Entre sus principales trabajos en temática europea, se pueden señalar: Lince Ibérico: Aspectos jurídicos para la conservación de la especie, Grupo Lince de la Sociedad Española para la Conservación y Estudio de los Mamíferos (SECEM) (Autora junto a Javier Calzada, Manuela Mora Ruiz, y Clara Márquez Ruíz), Málaga, ISBN 978-84-6143981-2, 2010; «La reunión de Copenhague de 2009: las nuevas negociaciones en torno al Principio de Responsabilidades Comunes pero diferenciadas de los Estados en materia de clima», Cuadernos Europeos de Deusto (ISSN 1130-8354), núm. 42 (Ejemplar dedicado a: Energía y Cambio Climático), pp. 125-153, 2010; Cambio Climático, Energía y Derecho Internacional: Perspectivas de Futuro (Coordinadora), Thomson Reuters Aranzadi, Pamplona (ISBN 978-84-9014-099-4), 2012; «Reflexiones en torno a la Jurisprudencia del Tribunal Europeo de Derechos Humanos en materia de igualdad de género», en La Praxis de la Paz y los Derechos Humanos. Joaquín Herrera Flores In Memoriam, Jesús Abellán Muñoz y otros-as (Editores), Eirene, Granada (ISBN 978-84-338-5439), pp. 263-285, 2012; «La acción internacional y europea en materia de cambio climático: construyendo la nueva fase 2013-2020», en La negociación de emisiones GEI en los mercados de carbono. Régimen y regulación, Isabel Rodríguez Martínez (Directora), Thomson Reuters Aranzadi, Pamplona (ISBN 978-84-9059-077-4), pp. 39-62, 2014. 


\section{About the author}

Rosa Giles Carnero is Professor of Public International Law and International Relations at the University of Huelva (Spain).

She has been coordinator of the Jean Monnet Module: European Union Climate Change Policy (2011-2014), concentrating his lines of research within the framework of European Law, on environmental and climate European policies, auropean action towards gender equality and environmental security. Among his main works on European themes are: Lince Ibérico: Aspectos jurídicos para la conservación de la especie, Grupo Lince de la Sociedad Española para la Conservación y Estudio de los Mamíferos (SECEM) (Autora junto a Javier Calzada, Manuela Mora Ruiz, y Clara Márquez Ruíz), Málaga, ISBN 978-84-614-3981-2, 2010; «La reunión de Copenhague de 2009: las nuevas negociaciones en torno al Principio de Responsabilidades Comunes pero diferenciadas de los Estados en materia de clima», Cuadernos Europeos de Deusto (ISSN 1130-8354), núm. 42 (Ejemplar dedicado a: Energía y Cambio Climático), pp. 125-153, 2010; Cambio Climático, Energía y Derecho Internacional: Perspectivas de Futuro (Coordinadora), Thomson Reuters Aranzadi, Pamplona (ISBN 978-84-9014-099-4), 2012; «Reflexiones en torno a la Jurisprudencia del Tribunal Europeo de Derechos Humanos en materia de igualdad de género», en La Praxis de la Paz y los Derechos Humanos. Joaquín Herrera Flores In Memoriam, Jesús Abellán Muñoz y otrosas (Editores), Eirene, Granada (ISBN 978-84-338-5439), pp. 263-285, 2012; «La acción internacional y europea en materia de cambio climático: construyendo la nueva fase 2013-2020», en La negociación de emisiones GEI en los mercados de carbono. Régimen y regulación, Isabel Rodríguez Martínez (Directora), Thomson Reuters Aranzadi, Pamplona (ISBN 97884-9059-077-4), pp. 39-62, 2014. 


\section{Derechos de autor}

Los derechos de autor (para la distribución, comunicación pública, reproducción e inclusión en bases de datos de indexación y repositorios institucionales) de esta publicación (Cuadernos Europeos de Deusto, CED) pertenecen a la editorial Universidad de Deusto. El acceso al contenido digital de cualquier número de Cuadernos Europeos de Deusto es gratuito inmediatamente después de su publicación. Los trabajos podrán leerse, descargarse, copiar y difundir en cualquier medio sin fines comerciales y según lo previsto por la ley; sin la previa autorización de la Editorial (Universidad de Deusto) o el autor. Así mismo, los trabajos editados en CED pueden ser publicados con posterioridad en otros medios o revistas, siempre que el autor indique con claridad y en la primera nota a pie de página que el trabajo se publicó por primera vez en $C E D$, con indicación del número, año, páginas y DOI (si procede). Cualquier otro uso de su contenido en cualquier medio o formato, ahora conocido o desarrollado en el futuro, requiere el permiso previo por escrito del titular de los derechos de autor.

\section{Copyright}

Copyright (for distribution, public communication, reproduction and inclusion in indexation databases and institutional repositories) of this publication (Cuadernos Europeos de Deusto, CED) belongs to the publisher University of Deusto. Access to the digital content of any Issue of Cuadernos Europeos de Deusto is free upon its publication. The content can be read, downloaded, copied, and distributed freely in any medium only for non-commercial purposes and in accordance with any applicable copyright legislation, without prior permission from the copyright holder (University of Deusto) or the author. Thus, the content of $C E D$ can be subsequently published in other media or journals, as long as the author clearly indicates in the first footnote that the work was published in $C E D$ for the first time, indicating the Issue number, year, pages, and DOI (if applicable). Any other use of its content in any medium or format, now known or developed in the future, requires prior written permission of the copyright holder. 